\title{
Dose-painting multicenter phase III trial in newly diagnosed glioblastoma: the SPECTRO-GLIO trial comparing arm A standard radiochemotherapy to arm $B$ radiochemotherapy with simultaneous integrated boost guided by MR spectroscopic imaging
}

Anne Laprie ${ }^{1,2^{*}}$ (D), Soléakhéna Ken ${ }^{2,3}$, Thomas Filleron ${ }^{4}$, Vincent Lubrano ${ }^{2,5}$, Laure Vieillevigne ${ }^{3}$, Fatima Tensaouti ${ }^{1,2}$, Isabelle Catalaa ${ }^{2,6}$, Sergio Boetto ${ }^{5}$, Jonathan Khalifa ${ }^{1}$, Justine Attal ${ }^{1}$, Guillaume Peyraga ${ }^{1}$, Carlos Gomez-Roca ${ }^{7}$, Emmanuelle Uro-Coste ${ }^{8}$, Georges Noel ${ }^{9}$, Gilles Truc ${ }^{10}$, Marie-Pierre Sunyach $^{11}$, Nicolas Magné ${ }^{12}$, Marie Charissoux ${ }^{13}$, Stéphane Supiot ${ }^{14}$, Valérie Bernier ${ }^{15}$, Muriel Mounier ${ }^{16}$, Muriel Poublanc ${ }^{16}$, Amandine Fabre ${ }^{16}$, Jean-Pierre Delord ${ }^{7}$ and Elizabeth Cohen-Jonathan Moyal ${ }^{1,17}$

\footnotetext{
Abstract

Background: Glioblastoma, a high-grade glial infiltrating tumor, is the most frequent malignant brain tumor in adults and carries a dismal prognosis. External beam radiotherapy (EBRT) increases overall survival but this is still low due to local relapses, mostly occurring in the irradiation field. As the ratio of spectra of choline/ $\mathrm{N}$ acetyl aspartate> 2 (CNR2) on MR spectroscopic imaging has been described as predictive for the site of local relapse, we hypothesized that dose escalation on these regions would increase local control and hence global survival.

Methods/design: In this multicenter prospective phase III trial for newly diagnosed glioblastoma, 220 patients having undergone biopsy or surgery are planned for randomization to two arms. Arm A is the Stupp protocol (EBRT 60 Gy on contrast enhancement $+2 \mathrm{~cm}$ margin with concomitant temozolomide (TMZ) and 6 months of TMZ maintenance); Arm B is the same treatment with an additional simultaneous integrated boost of intensity-modulated radiotherapy (IMRT) of 72Gy/2.4Gy delivered on the MR spectroscopic imaging metabolic volumes of CHO/NAA > 2 and contrast-enhancing lesions or resection cavity. Stratification is performed on surgical and MGMT status.

Discussion: This is a dose-painting trial, i.e. delivery of heterogeneous dose guided by metabolic imaging. The principal endpoint is overall survival. An online prospective quality control of volumes and dose is performed in the experimental arm. The study will yield a large amount of longitudinal multimodal MR imaging data including planning $C T$, radiotherapy dosimetry, MR spectroscopic, diffusion and perfusion imaging.

(Continued on next page)
}

\footnotetext{
* Correspondence: laprie.anne@iuct-oncopole.fr

${ }^{1}$ Radiation Oncology Department, Institut Claudius Regaud- Institut

Universitaire du Cancer de Toulouse-Oncopole, Toulouse, France

${ }^{2}$ ToNIC, Toulouse Neurolmaging Center, Université de Toulouse, INSERM,

UPS, Toulouse, France

Full list of author information is available at the end of the article
}

(c) The Author(s). 2019 Open Access This article is distributed under the terms of the Creative Commons Attribution 4.0 International License (http://creativecommons.org/licenses/by/4.0/), which permits unrestricted use, distribution, and reproduction in any medium, provided you give appropriate credit to the original author(s) and the source, provide a link to the Creative Commons license, and indicate if changes were made. The Creative Commons Public Domain Dedication waiver (http://creativecommons.org/publicdomain/zero/1.0/) applies to the data made available in this article, unless otherwise stated. 
(Continued from previous page)

Trial registration: NCT01507506, registration date December 20, 2011.

Keywords: Glioblastoma, Dose-painting, Magnetic resonance spectroscopic imaging, Spectroscopy, Proton spectroscopy, Radiotherapy, Clinical trial, Phase III,online prospective quality control,

\section{Background}

Glioblastoma (GBM) is an infiltrating heterogeneous brain tumor characterized by high cellular proliferation, high cellular density and active angiogenesis associated with areas of necrosis. After surgery or biopsy, irradiation is indicated as it improves overall survival, although most patients present local relapse in the irradiation fields. Prognosis is therefore dismal with a median overall survival of 8 to 14 months [1]. The failure to achieve sustainable local control in this tumor emphasizes the need to develop innovative treatment strategies. An attractive approach is to define new RT target volumes that include active disease, which can be highlighted by functional imaging. A promising strategy to tackle this radioresistance and attempt to improve local control consists of irradiating the target volume heterogeneously, with focal increases in dose targeted at radioresistant clusters defined by metabolic imaging $[2,3]$. This 'dose-painting' approach targets metabolic abnormalities that are not only prognostic indicators of aggressiveness but are also predictive of local relapse after treatment.

In this context, in vivo ${ }^{1} \mathrm{H}$ magnetic-resonance spectroscopic imaging (MRSI) has shown significant promise [4-7]. It measures the concentration and spatial distribution of tissue metabolites like choline (Cho) and $\mathrm{N}$-acetyl-aspartate (NAA) which are respectively membrane and neuronal markers. An elevated Cho/NAA ratio (CNR) indicates increased cellular proliferation and reduced neuron density, and is assumed to highlight a metabolically active part of the tumor in high-grade gliomas $[5,7]$. This metabolic tool is a useful predictor of survival $[8,9]$ and relapse location in GBM patients [6].

Our team showed [4] that magnetic resonance spectrometric imaging (MRSI) is useful in depicting areas with a high potential for recurrence. In that study, 23 reviews were conducted for 9 patients studied in a phase I associating tipifarnib and radiotherapy. Patients underwent MRI and MRSI before treatment and every 2 months after RT until relapse. The MRSI data were categorized by the choline (Cho) / N-acetyl-aspartate (NAA) ratio (CNR) to measure spectroscopic abnormalities. The study, which correlated spectral and morphologic abnormalities prior to radiotherapy with the same data at the time of relapse for 1207 voxels, showed that the volumes of spectral anomalies were more limited than morphological abnormalities. In fact, before radiotherapy, the CNR $>2$ regions accounted for $25 \%$ of contrast regions and $10 \%$ of regions of T2 hyperintensity (excluding contrasting volumes). The presence of metabolically active regions was predictive of the site of relapse after radiotherapy. Indeed, $75 \%$ of the contrast enhanced lesion $(\mathrm{CE})+\mathrm{CNR} 2$ regions before radiotherapy continued to be CNR2 at relapse, compared to $22 \%$ of voxels with a normal CNR before RT $(p<0.05)$.

Several recent studies have investigated different methods for increasing the dose delivered to glioblastomas. Although previous studies showed only the feasibility of escalating doses without benefit but without toxicity, the most recent findings found a benefit in selected populations.

In 2004, Cho et al. [10] compared the modalities of boost after conformational radiotherapy (RTC) (60 Gy / $2 \mathrm{~Gy}$ ) delivered at an average 1.4 months after the end of RTC; 14 patients received a boost in radiosurgery with a median fraction of $10.5 \mathrm{~Gy}(10-18)$ and 10 others received a boost in stereotactic fractionated radiotherapy for a median dose of 27.5 Gy (20-35) in 11 fractions. There was an improvement in survival rates with fewer complications in the fractional stereotactic boost group. In 2004 Sultanem et al. [11] published a study of irradiation in intensity modulation in 20 fractions: $60 \mathrm{~Gy} /$ $3 \mathrm{~Gy}$ on the GTV and $40 \mathrm{~Gy} / 2 \mathrm{~Gy}$ on the PTV (GTV + $1.5 \mathrm{~cm}$ ). Tolerance was good but survival was not modified. In 2002, Nwokedi et al. [12] reported a retrospective study comparing 33 patients who received $60 \mathrm{~Gy} /$ 2 Gy with 31 patients who received gamma-knife radiosurgery of $17.1 \mathrm{~Gy}$ on average (10-28); in the group receiving the boost, survival was 25 months versus 13 months in the other group. Tolerance was identical. Tanaka et al. [13] showed that 80 Gy boosts of conformal external radiotherapy on contrast enhancement could lead to an increase in survival. However, these results need to be confirmed since there was no identical comparison group but only an historical comparison group. The more robust study by Cardinale et al. published in 2006 [14] reported the results of the RTOG0023 multicenter trial on 76 patients who received 50 Gy per 2 Gy fractions and a weekly stereotactic boost of 5 or $7 \mathrm{~Gy}$, resulting in a dose equivalent of 70 or $78 \mathrm{~Gy}$ in 6 weeks. The treatment was feasible and well tolerated with a benefit only for patients with macroscopically complete resection. Of the 65 patients suspected of progression, half were re-operated: $15 \%$ had only necrosis and $85 \%$ had tumor necrosis. 
These interesting dose-increase studies in newly diagnosed glioblastomas showing positive effects on survival did included neither concomitant temozolomide nor metabolic imaging to guide boost delivery, nor a concomitant daily boost included in the initial radiotherapy treatment. This prompted us to test concomitant radiochemotherapy with an integrated boost targeted at metabolic predictive abnormalities. We calculated the equivalent integrated boost of $72 \mathrm{~Gy} / 2.4 \mathrm{~Gy}$ corresponding to the sequential boosts that had yielded survival improvement.

\section{Material and method}

\section{Study design (Fig. 1)}

The trial is a multicenter two-armed randomized phase III study.

Patients fulfilling the inclusion criteria are randomized with computer-generated random numbers. Stratification is performed on surgical and MGMT status. The two arms are:

\section{Arm a: Standard arm}

3D conformal radiotherapy or IMRT delivering 60 Gy per fraction of $2 \mathrm{~Gy}$ in 30 fractions on contrast-enhancing lesions or tumor bed $+2 \mathrm{~cm}$, with concomitant TMZ and 6 months maintenance of TMZ.

\section{Arm B: Experimental arm}

IMRT delivering 60 Gy per fraction of 2 Gy in 30 fractions on contrast-enhancing lesions or tumor bed +2 $\mathrm{cm}$, and $72 \mathrm{GY} / 2.4 \mathrm{~Gy}$ with a simultaneous integrated boost guided at MRSI $\mathrm{CHO} / \mathrm{NAA}>2+10 \mathrm{~mm}$ and tumor bed $+3 \mathrm{~mm}$ with concomitant TMZ and 6 months maintenance of TMZ.

\section{Study objectives}

Primary objective is overall survival. Secondary objectives are as follows: event-free survival; secondary effects of dose intensification, particularly intracranial hypertension, late effects such as radiation necrosis, increase in epileptic seizures; evaluation of individual, imaging and biologic markers associated with overall survival, eventfree survival and sites of local relapse.

\section{Patient selection: Inclusion criteria}

- Age $>18$ years

- PS $\leq 2$

- Glioblastoma

- Biopsy or surgery

- Methylation status of MGMT gene

- In the event of surgery, the patient must have undergone an early MRI or CT scan to assess the presence of macroscopic residue.

- Surgery or biopsy performed in a maximum of 45 days before the first radiotherapy fraction. Randomization must be performed in the 32 days after surgery /biopsy to allow centralized contouring, dosimetry calculation and online prospective quality control.

- Written informed consent

\section{Patient selection: exclusion criteria}

- Impossibility to analyze MRSI metabolic maps, especially in the event of massive post-surgery hemorrhage inducing artefacts.

- Multifocal glioblastoma

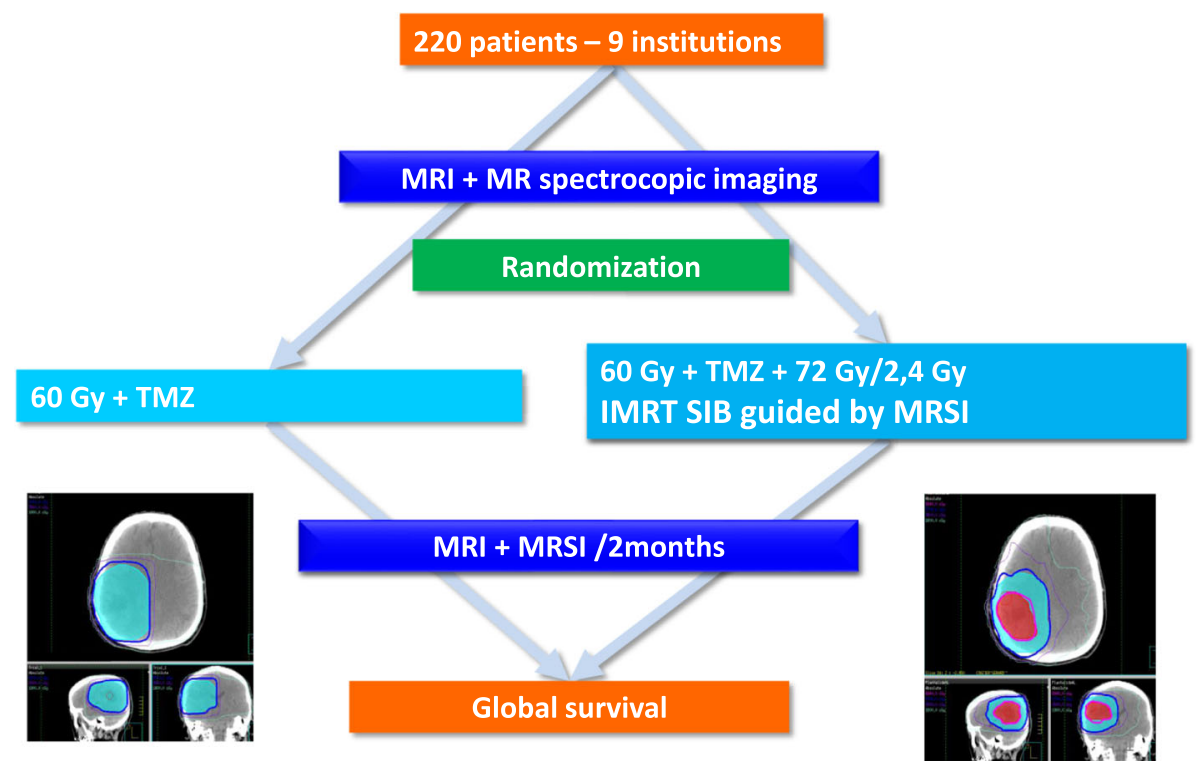

Fig. 1 Study design 
- Leptomeningeal metastasis

- Epileptic attack despite anticonvulsant treatment

- Previous chemo- or radiotherapy to treat glioblastoma

- Abnormalities of blood cell count if neutrophils < $1500 / \mathrm{mm}^{3}\left(1.5 \times 10^{9} / \mathrm{l}\right)$ and platelets $<100,000 / \mathrm{mm}^{3}$ $\left(100 \times 10^{9} / 1\right)$

- Renal insufficiency

- Refusal to participate

- Previous re-irradiation or previous radiosurgery

- Previous treatment with interstitial radioactive seeds

- Carcinoma known < 5 years ago (excluding carcinoma in situ of the cervix, basal cell

carcinoma, squamous cell carcinoma of the skin) requiring immediate treatment interfering with study therapy.

- Pregnancy or lactation.

- Participation in another clinical trial

- Contraindications for MRI examination (e.g. claustrophobia, pacemaker)

- Disorder precluding understanding of trial or informed consent

- Diameter $>5 \mathrm{~cm}$

- Distance from chiasma to tumor bed or MR spectroscopic abnormalities $2 \mathrm{~cm}$

\section{Imaging and radiotherapy requirements Imaging requirements}

In this multicenter spectroscopy trial, the reproducibility and reliability of the technique are based on criteria for placement of the volume of interest, post-processing of spectral acquisition, quantification, and creation of metabolic maps. Findings to date have only been monocenter $[4,7$, 15]. To ensure reliable and comparable post-processing between all centers, in the absence of "universal" spectrometry-processing software, all examinations are carried out on MRIs equipped with the CSI 3D module from the same manufacturer on a $1.5 \mathrm{~T}$ MRI scan (Siemens, Erlangen, Germany). Before inclusion began, each center performed an MRI + ISRM examination for 4 patients with glioblastoma according to the modalities defined for this protocol and sent the data to the promoter. Before the start of the trial, the coordinating center evaluated the quality of the images and spectra acquired. A team from the coordinating center including the coordinating radiation oncologist, the imaging-radiotherapy engineer and a physicist visited each center prior to the trial. This allowed any necessary modifications or improvements to be made before the trial began.

\section{Radiotherapy requirements}

The participating centers are academic or private centers, they must be able to use IMRT. Each center must perform an internal quality control of the accelerator. Furthermore, the calibration of the beams used in this study (external quality control) must comply with the requirements laid down in the technical decision of the AFSSAPS, the French health and safety agency (published 2 March 2004, updated 27 July 2007).

\section{Selection and randomization (Fig. 2)}

After screening by the referring radiation oncologist, patients first undergo a multimodal MRI including T1 without and with gadolinium, FLAIR, diffusion-weighted sequences (DWI), perfusion-weighted imaging sequences (PWI) and 3D CSI MR spectroscopic imaging. Participating centers use a dedicated web-based database (equal ESTRO) to send MRI and spectroscopy post-processing data to the coordinating center. The MR spectroscopic imaging of CNR2 is obtained after analysis of the MR spectroscopy (Fig. 3). The size of the tumor and CNR2 must be $<5 \mathrm{~cm}$ and the tumor bed and CNR2 must be $>2 \mathrm{~cm}$ from the chiasma. Patients are then randomized.

\section{Treatment planning}

For arm B, all contours, including target volumes and organs at risk (OAR), are assessed by the coordinating center and sent to the participating center. Regarding technical difficulties for planning treatment, there are two main issues for including MRSI in a RT treatment planning system (TPS). Firstly, MRSI images obtained from MRI scanners do not conform to DICOM standards (DICOM 3.0) and are MR spectroscopic maps overlaid on corresponding anatomical MR images. These images, and unlike conventional MR images are not compatible for automatic image fusion with planning CT scans. Secondly, the escalation in radiation dose from the simultaneous integrated boost (SIB) should be carefully evaluated, especially for organs at risk (OAR).

We therefore performed a preliminary study to address these two issues and published [16] a method for incorporating metabolic maps into TPS, overcoming the absence of a DICOM 3.0 standard for MRSI, to guide the simultaneous integrated boost. In the same paper, we compared dosimetry plans of the standard $60 \mathrm{~Gy}$ treatment in 3D conformational radiotherapy (60-Gy 3D-CRT), 60 Gy in IMRT and treatment with the dose escalation of 72 Gy in SIB-IMRT. When comparing the dose received by OAR, there was no significant difference between 60-Gy 3D-CRT, 60 Gy IMRT and 72 Gy SIB-IMRT: i.e. there was no difference in the maximum dose to the optic chiasm and the mean dose to the normal brain. Compared to 60 Gy 3D-CRT, both $60-$ Gy IMRT and 72-Gy SIB-IMRT significantly lowered the dose to the brainstem. The method is used in this trial 


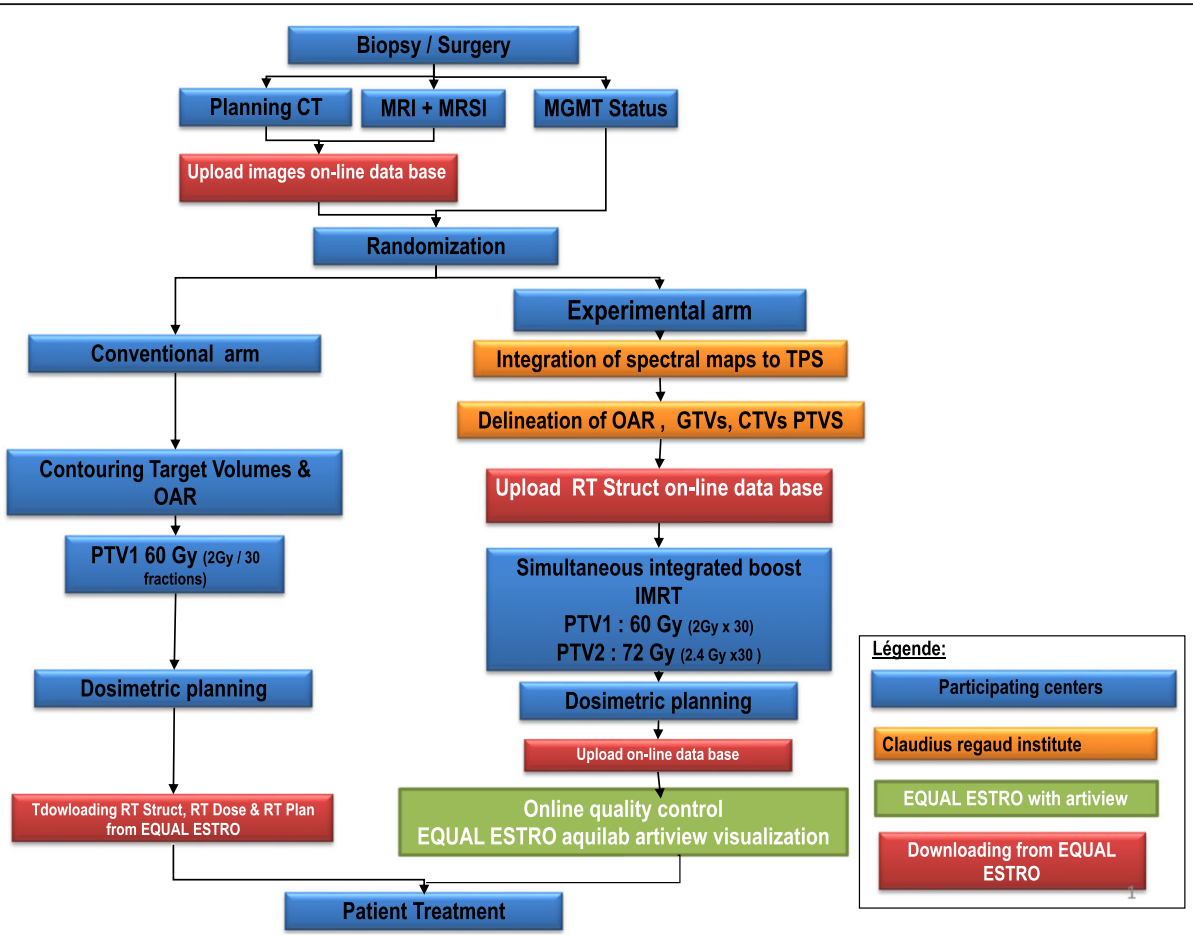

Fig. 2 Study workflow

for including MR spectroscopic imaging of Cho/NAA > 2 when planning radiotherapy treatments.

\section{Dose prescription (Fig. 4 and 5)}

Patients are treated in one of two arms. For the two treatment arms (definition on pre-RT MRI): GTV1: contrast enhancement on MRI pre-RT if biopsy, or if surgery on the operative bed and possible residual contrast on pre-RT MRI, CTV 1: GTV + $17 \mathrm{~mm}$ including $\mathrm{T} 2$ signal abnormalities, then corrected to remove bone and air.. PTV 1: CTV1+3 mm. No manual changes to PTV1 should be made.

For Arm B, the GTV2, CTV2 and PTV2 are defined in addition to the following criteria: GTV2: Region defined in magnetic resonance spectrometry as the region with high relapse potential presents a signal finding Cho / NAA > 2 CTV2: GTV2 + $7 \mathrm{~mm}+\mathrm{GTV} 1$ by correcting it to eliminate bone, air or other structure that cannot be extended. If CTV2 (presence of spectral abnormalities) is not included in CTV1, then CTV1 is expanded to include CTV2.

PTV2: CTV2 $+3 \mathrm{~mm}$. No manual changes to PTV2 should be made. Delineated organs at risk are optic nerve, brainstem, optic chiasm, posterior chamber of eye, anterior chamber of eye, supra-tentorial brain.

PTV 2 receives a daily dose of 2.4 Gy for a cumulative dose of 72 Gy. Only irradiation with concomitant boost ( $\mathrm{SIB}=$ simultaneous integrated boost $)$ is permitted so

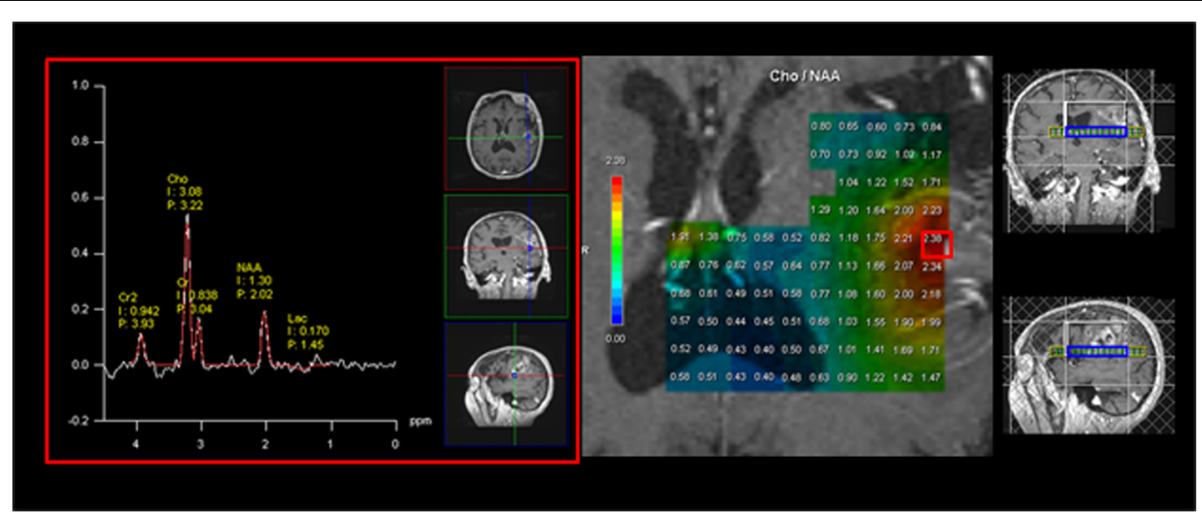

Fig. 3 Example of an arm B patient:3A MRSI map showing abnormalities of Cho/NAA > 2 


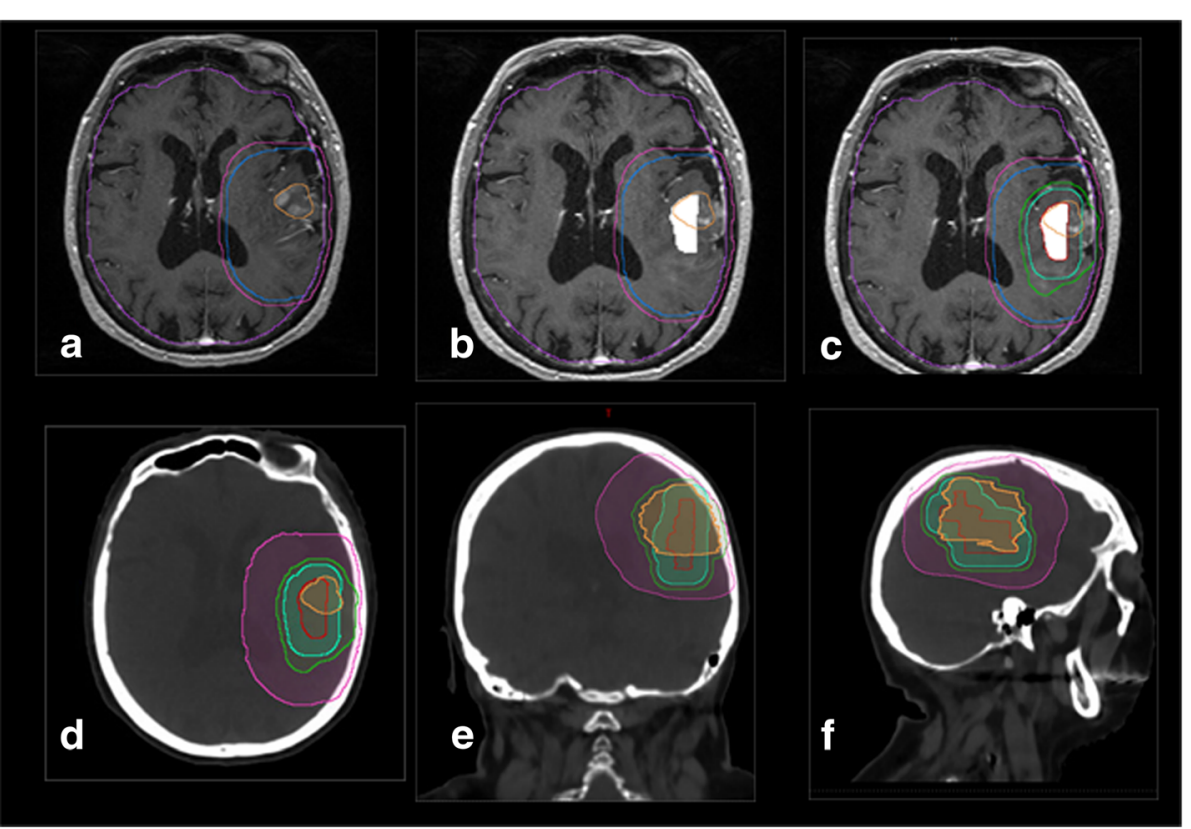

Fig. 4 a-delineated GTV1 (orange), CTV1 (blue), PTV1 (pink) on T1gado MRI, 4b MRSI map, 4Bc GTV1, CTV1, PTV1 GTV2(red), CTV2(cyan), PTV2 (green) 4 d resulting volumes on axial view (d), frontal view (e) sagittal view (f)

sequential irradiation is not allowed. Irradiation is delivered in static, dynamic or arc intensity-modulated RT on linear accelerator or on tomotherapy. In each arm, treatment is associated with TMZ for 6 months after radiotherapy according to the standard Stupp protocol.

\section{Dose criteria for target volumes}

The PTV prescribing criteria must follow the recommendations of ICRU 83:

- 95\% of the PTV should receive at least $95 \%$ of the dose

- 98\% of the PTV should receive at least $90 \%$ of the dose
- 3\% of the PTV should not receive more than 107\% of the dose

- prescribed dose at the median of the PTV. In treatment arm B (with integrated boost), the prescribed doses are 60 Gy for PTV1 and 72 Gy for PTV2. Dose criteria for risk organs dose-volume histograms are generated for PTV, brain PTV, visual structures and brainstem.

During radiotherapy, temozolomide (TMZ) is administered orally at a dose of $75 \mathrm{mg} / \mathrm{m} 2$ / day, from the first day to the last day of radiation therapy, including Saturdays, Sundays and public holidays [17]. At the end of radiotherapy, the treatment with TMZ is stopped and then begins 4 weeks after the end of the radiotherapy

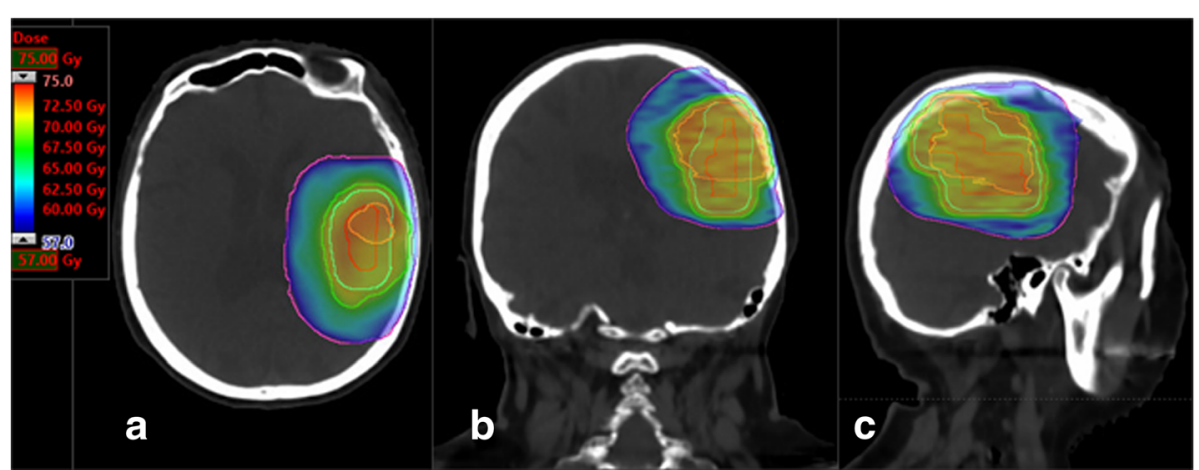

Fig. 5 Corresponding dosimetry in a) axial slice, b) sagittal orientation, c) coronal orientation, GTVs, CTVs and PTVs are displayed with identical color lines as in Fig. 4. The isodoses are displayed in colorwash. In Blue 95\% of 60Gy on PTV1, in orange 95\% of 72Gy on PTV2 
according to the following modalities: temozolomide (TemodalR): Orally. - $150 \mathrm{mg} / \mathrm{m} 2 /$ day for 5 days for the first cycle (from D1 to D5) - resumed at D 28 (D28 to D32) and then every 28 days at $200 \mathrm{mg} / \mathrm{m} 2$ / day for 5 days if the hematological tolerance of the first cycle was good (in total 6 cycles of 5 days of adjuvant TMZ).

\section{Imaging data acquisition}

Before inclusion and at each follow-up, patients undergo an MRI scan that includes: anatomical T1-weighted sequences, before and after contrast injection, FLAIR, T2, 3D MRSI, perfusion weighted imaging (PWI) with dynamic susceptibility contrast (DSC) and diffusionweighted imaging (DWI). All scans in each center are performed on a Siemens 1.5-T MRI scanner (Siemens, Erlangen, Germany) with a standard 12-channel head coil. Parallel imaging acquisition is used for each patient, based on the GRAPPA (Generalized autocalibrating partially parallel acquisition) reconstruction algorithm, with an acceleration factor of 2 . The anatomical MRI protocol includes: acquisition of $3 \mathrm{~mm}$-thick axial images (turbo spin-echo T2-weighted imaging (TR/TE $=4200 / 97 \mathrm{~ms}$ ), (FLAIR: TR/TI $/ \mathrm{TE}=6500 / 2400 / 121 \mathrm{~ms}$ )) with a field of view (FOV) of $172 \times 230 \mathrm{~mm}^{2}$ and a matrix size of $256 \times$ 192 , resulting in a voxel size of $0.9 \times 0.9 \times 3 \mathrm{~mm}^{3}$; and acquisition of $1 \mathrm{~mm}$-thick 3D T1-weighted images (T1) before and after injection of a standard dose of $15 \mathrm{ml}$ of gadolinium-based contrast agent (Gadobenate dimeglumine, MultiHance ${ }^{\circ}(\mathrm{T} 1-\mathrm{Gd}: \mathrm{TR} / \mathrm{TE}=11 / 5.2 \mathrm{~ms}, \mathrm{FOV}=$ $256 \times 224$, matrix size $=256 \times 224$, resulting in a voxel size of $1 \times 1 \times 1 \mathrm{~mm}^{3}$ ).

3D-chemical-shift imaging (3D-CSI) for MRSI acquisition consists of three phase-encoded gradients prior to read-out, resulting in a scan time of $8 \mathrm{~min}$. MRSI acquisition consists of a spin-echo-based sequence with the following parameters: TR/TE $=1500 \mathrm{~ms} / 135 \mathrm{~ms}$ for lactate detection, and four excitations. FOV is set by default at $100 \times 100 \mathrm{~mm}^{2}$ for a CSI matrix of $16 \times 16$, with eight slices of $25.0 \mathrm{~mm}$ thickness, resulting in a voxel resolution of $6.26 \times 6.25 \times 25.0 \mathrm{~mm}^{3}$, i.e. $1 \mathrm{~cm}^{3}$. Adjustments on the 3D-CSI box are performed when needed to cover entirely or the majority of abnormalities and when possible normal-appearing tissue, while avoiding regions that could corrupt the spectra such as bone and subcutaneous lipids. Saturation bands are also positioned around the volume of interest (VOI) to suppress signals from excited regions outside the VOI, and to provide good in vivo fat suppression. Manual shimming is performed to reach a line width $<15 \mathrm{~Hz}$.

For DSC-MRI, a series of 34 volumes of 39 slices are acquired at 1.09-s intervals, with a gradient-echo echo-planar imaging sequence $(\mathrm{TR} / \mathrm{TE}=4720 / 47 \mathrm{~ms}, \mathrm{FOV}=230 \times 230$ $\mathrm{mm}^{2}$, matrix size $=128 \times 128$, flip angle $\left.=90^{\circ}\right)$, during the first pass of a standard dose $(0.1 \mathrm{mmol} / \mathrm{kg})$ bolus of gadolinium. Gadolinium is injected intravenously using a power injector at a rate of $5 \mathrm{~mL} / \mathrm{s}$, immediately followed by a saline injection. DWI is performed with a single-shot, spin-echo, echo-planar imaging sequence in the axial plane $(\mathrm{TR} / \mathrm{TE}=8300 / 91 \mathrm{~ms}$ at $\mathrm{b}=0$ and $\mathrm{b}=1000 \mathrm{~s} / \mathrm{mm} 2,25-40$ sections, 2 -mm section thickness, FOV $=230 \times 230 \mathrm{~mm}^{2}$, matrix size $=192 \times 192$ ). DWI is acquired in three orthogonal directions. CT simulation images for RT planning of all patients are acquired in helical mode with a voxel resolution of $0,98 \times 0.98 X 2.5 \mathrm{~mm}^{3}$.

\section{Spectroscopic data processing}

The spectroscopic processing protocol consists of water subtraction, low-pass filtering, frequency shift correction, baseline correction, phase correction and curve-fitting in the frequency domain. These steps of spectra processing are performed with the Siemens Syngo MR B17 spectroscopy application (Erlangen, Germany).

\section{Quality control}

A dummy run study is performed in both arms, both for MR spectroscopic acquisition and for arm A contouring + dosimetry as well as dosimetry for arm B.

Online contouring for arm B.

Online control quality of dosimetry for arm B.

This procedure is described in Fig. 2 and the delay for starting radiotherapy the latest at 42 days after biopsy or surgery must be respected. Then the dosimetry is performed in the participating center and arm B provisional dosimetries undergo prospective online quality control performed by an independent physicist and by an independent radiation oncologist reviewer.

\section{Treatment schedule}

The treatment must start within 45 days after biopsy or surgery. The fractions should be delivered within 42 days (6 weeks) and the total duration of treatment must not exceed 48 days. A bi-fractionation ( 2 fractions delivered the same day at more than $6 \mathrm{~h}$ interval) is allowed a maximum of three times during the treatment and not more than once a week. The patient receives 5 sessions maximum per week, bi-fractionations included. Treatment interruptions must be clearly recorded in the treatment record as well as the reasons for the interruptions. There must be no more than 3 consecutive interruption working days and a maximum of 6 days in total. Interruptions are tolerated only for medical reasons due to severe side effects or other concomitant diseases, but not for social or logistical reasons.

\section{Follow-up Clinical follow-up}

Patients undergo medical examination every week during radiotherapy and every 21 days during maintenance temozolomide. 


\section{Imaging procedures}

MRI (including $\mathrm{T} 1$ without and with gadolinium enhancement, T2, FLAIR, PWI(DSC), DWI and MRSI (3D-CSI) examinations are carried out as follows:

- 1st examination 3 months after the end of radiotherapy in order to have the maximum effect of radiotherapy and to avoid aspects of pseudo-progression [18], then every 2 months. In the interest of the patient, if the patient shows suspicious clinical signs within 3 months after the end of radiotherapy, MRI and MRSI control are performed earlier. Due to the risk of pseudo-progression, a new MRI + MRSI is performed 1 month later in order to verify (to check) the reality of the progression (for any image of progression or new contrast in radiation field during the first MRI of control) [18]. In the event of stability or regression, the patient is considered as non-relapsing.

\section{Definition of event}

Relapse is defined with the RANO criterias [19]. In the event of relapse, treatment is decided upon by the investigator.

Adverse events and other unintended effects of trial interventions or trial conduct this events are collected by the research technicians, reported and an external security toxicity audit is performed every year.

\section{Statistical analysis}

The main objective is to increase 2-year overall survival from 25\% (standard arm "radiotherapy plus temozolomide") to $40 \%$ in the experimental arm. This hypothesis corresponds to detecting a hazard ratio of 0.66 . A total of 186 deaths are necessary for $80 \%$ power to detect this difference if it is true using a two-sided logrank test at the $5 \%$ level of significance and a 1:1 randomization. Based on an estimated accrual rate of approximately 70 patients per year and a fixed follow-up of 3 years, 220 patients need to be included. An interim analysis for efficacy will be performed after 93 events have been observed (Lan deMets O’Brien Fleming Boundaries).

\section{Discussion}

The SPECTRO GLIO trial is one of the few ongoing dose-escalation or dose-painting trials in Europe [20-24]. It is based on the value of an advanced type of metabolic imaging to predict the site of relapse for glioblastoma after radiotherapy, i.e. $\mathrm{CHO} / \mathrm{NAA}$ ratio $>2$. MR spectroscopic imaging is used as a target. The use of SIB makes it possible to increase significantly the dose without increasing the dose to organs at risk [16].

A strength of the trial is the centralized analysis of MR spectroscopy, the centralized contouring for the experimental arm, and the online prospective quality control of dosimetry in the experimental arm. As described for several trials $[25,26]$ and in two recent metaanalysis $[27,28]$, we strongly believe that quality control and homogeneity of treatment are crucial to provide reliable results in this type of trial. By February 2018, 180 patients had been included and the intermediate analysis is planned after 93 events.

The issue of glioblastoma dose-painting is a shared interest as a comparable phase II trial with a 75 Gy boost targeted at CNR > 2 started in September 2017 in the US (NCT03137888) as a pilot study in 36 patients with the use of whole brain MR spectroscopy. The SPECTRO GLIO trial includes a large amount of imaging data and dosimetry information: planning $\mathrm{CT}$, all contours of organs at risks and target volumes, longitudinal $\mathrm{T} 1$, FLAIR, diffusion and perfusion MRI, as well as 3D MR Spectroscopic imaging. This large longitudinal set of data will provide useful information on the natural history of glioblastoma correlated with different levels of radiotherapy dose. We have already performed imaging studies on subsets of patients included in this trial $[29,30]$ and will carry out further studies on the whole cohort, as this may lead to new prognostic and predictive values for anatomic and metabolic imaging, particularly the predictive value of $\mathrm{CHO} / \mathrm{NAA}$ and lactates/NAA [31] already published in small subsets of patients.

\section{Abbreviations}

3D-CRT: Three-dimensional conformation radiation therapy; CE: contrast enhancement; CSI: chemical Shift imaging; DSC: Dynamic Susceptibility contrast; DWI: Diffusion Weighted Imaging; FLAIR: Fluid Attenuated Inversion Recovery; GBM: glioblastoma; IMRT: intensity Modulated Radiotherapy; MRI: magnetic resonance imaging; MRSI: Magnetic Resonance Spectroscopic Imaging; PWI: Perfusion Weighted Imaging; SIB: Simultaneous Integrated Boost; TMZ: Temozolomide

\section{Acknowledgements}

We thank Anne-Laure Fize and Ray Cooke for English editing.

\section{Trial sponsor}

Institut Claudius Regaud.

Institut Universitaire du Cancer de Toulouse -oncopole, 1 avenue Irène JoliotCurie, F-31059 Toulouse.

Contact Poublanc.muriel@iuct-oncopole.fr

\section{Funding}

This trial is funded entirely by the French National Institute for Cancer. The funding body has no role in the design of the study and collection, analysis, nor interpretation of data nor writing the manuscript.

Availability of data and materials

Data sharing is not applicable to this article.

\section{Authors' contributions}

AL coordinates the trial, wrote the manuscript. AL and SK prepared the Figs. $A L, S K, L V$, TF participated in the manuscript writing. AL, ECJM, SK, LV, IC, TF, MP elaborated the trial design. AL, SK, ECJM, JK, SB, VL, CGR, GT, GN, MPS, $M C, N M, G P, J A, M M, A F, E U C, J P D$ participated in the preparation of the patient inclusion, treatment and follow-up workflow. ECJM, JK, SB, VL, CGR, GT, GN, MPS, MC, NM, GP, JA, MM, AF, EUC, JPD participated in the preparation of the patient inclusion, treatment and follow-up workflow. AL, SK and IC elaborated and set-up the imaging workflow. AL, VB, SS, MM, AF 
participated in the elaboration and set-up of the prospective online quality control. All authors read and approved the final manuscript.

\section{Ethics approval and consent to participate}

This trial was reviewed and approved by the French ethics committee on 28 April 2010: registration number 2009-A00594-53.

This covers all participating centers.which are:

Institut Claudius Regaud at Institut Universitaire du Cancer de Toulouse-

Oncopole- Toulouse-France.

Centre Paul Strauss- Strasbourg- France.

Centre Georges-François Leclerc - Dijon- France.

Centre Léon Bérard- Lyon- France.

Institut de Cancérologie de la Loire- Saint-Priest en Jarez.

Centre Val d'aurelle - Montpellier- France.

Clinique Claude Bernard- Albi- France.

All subjects signed a written informed consent form.

\section{Consent for publication}

Consent for publication is not applicable to this article.

\section{Competing interests}

The authors declare that they have no competing interests.

\section{Publisher's Note}

Springer Nature remains neutral with regard to jurisdictional claims in published maps and institutional affiliations.

\begin{abstract}
Author details
${ }^{1}$ Radiation Oncology Department, Institut Claudius Regaud- Institut Universitaire du Cancer de Toulouse-Oncopole, Toulouse, France. ${ }^{2}$ ToNIC, Toulouse Neurolmaging Center, Université de Toulouse, INSERM, UPS, Toulouse, France. ${ }^{3}$ Department of Engineering and Medical Physics, Institut Claudius Regaud- Institut Universitaire du Cancer de Toulouse-OncopoleCancer de Toulouse-Oncopole, Toulouse, France. ${ }^{4}$ Biostatistics Unit, Institut Claudius Regaud- Institut Universitaire du Cancer de Toulouse-Oncopole, Toulouse, France. ${ }^{5}$ Neurosurgery Department, Centre Hospitalier Universitaire de Toulouse, Toulouse, France. ${ }^{6}$ Neuroimaging Department, Centre Hospitalier Universitaire de Toulouse, Toulouse, France. ${ }^{7}$ Medical Oncology Department, Institut Claudius Regaud- Institut Universitaire du Cancer de Toulouse-Oncopole, Toulouse, France. ${ }^{8}$ Pathology department, Centre Hospitalier Universitaire de Toulouse, Toulouse, France. ${ }^{9}$ Radiation Oncology Department, Centre Paul Strauss, Strasbourg, France.

${ }^{10}$ Radiation Oncology Department Centre Georges-François Leclerc, Dijon, France. ${ }^{11}$ Radiation Oncology Department- Centre Léon Bérard, Lyon, France. ${ }^{12}$ Radiation Oncology Department, Institut de Cancérologie de la Loire, Saint-Priest en Jarez, France. ${ }^{13}$ Radiation Oncology Department - Centre Val d'aurelle, Montpellier, France. ${ }^{14}$ Radiation Oncology Department, Institut de Cancerologie de l'Ouest, Nantes st Herblain, France. ${ }^{15}$ Radiation Oncology Department, Institut de cancérologie de Lorraine centre Alexis Vautrin, Nancy, France. ${ }^{16}$ Clinical Research Department, Institut Claudius Regaud, Institut Universitaire du Cancer de Toulouse-Oncopole, Toulouse, France. ${ }^{17}$ INSERM UMR1037, Cancer Research Center of Toulouse, Oncopole, Toulouse, France.
\end{abstract}

Received: 19 May 2018 Accepted: 24 January 2019

Published online: 21 February 2019

\section{References}

1. Johnson DR, O'Neill BP. Glioblastoma survival in the United States before and during the temozolomide era. J Neuro-Oncol. 2012;107:359-64.

2. Ling CC, Humm J, Larson S, Amols H, Fuks Z, Leibel S, et al. Towards multidimensional radiotherapy (MD-CRT): biological imaging and biological conformality. Int J Radiat Oncol Biol Phys. 2000;47:551-60.

3. Bentzen SM, Gregoire V. Molecular imaging-based dose painting: a novel paradigm for radiation therapy prescription. Semin Radiat Oncol. 2011;21:101-10

4. Laprie A, Catalaa I, Cassol E, McKnight TR, Berchery D, Marre D, et al. Proton magnetic resonance spectroscopic imaging in newly diagnosed glioblastoma: predictive value for the site of Postradiotherapy relapse in a prospective longitudinal study. Int J Radiat Oncol Biol Phys. 2008;70:773-81.
5. McKnight TR, von dem Bussche MH, Vigneron DB, Lu Y, Berger MS, McDermott MW, et al. Histopathological validation of a three-dimensional magnetic resonance spectroscopy index as a predictor of tumor presence. J Neurosurg. 2002;97:794-802.

6. Park I, Tamai G, Lee MC, Chuang CF, Chang SM, Berger MS, et al. Patterns of recurrence analysis in newly diagnosed glioblastoma multiforme after threedimensional conformal radiation therapy with respect to pre-radiation therapy magnetic resonance spectroscopic findings. Int J Radiat Oncol Biol Phys. 2007:69:381-9.

7. Pirzkall A, Li X, Oh J, Chang S, Berger MS, Larson DA, et al. 3D MRSI for resected high-grade gliomas before RT: tumor extent according to metabolic activity in relation to MRI. Int J Radiat Oncol Biol Phys. 2004; 59:126-37.

8. Crawford FW, Khayal IS, McGue C, Saraswathy S, Pirzkall A, Cha S, et al. Relationship of pre-surgery metabolic and physiological MR imaging parameters to survival for patients with untreated GBM. J Neuro-Oncol. 2009;91:337-51.

9. Saraswathy S, Crawford FW, Lamborn KR, Pirzkall A, Chang S, Cha S, et al. Evaluation of MR markers that predict survival in patients with newly diagnosed GBM prior to adjuvant therapy. J Neuro-Oncol. 2009;91:69-81.

10. Cho KH, Hall WA, Lo SS, Dusenbery KE. Stereotactic radiosurgery versus fractionated stereotactic radiotherapy boost for patients with glioblastoma multiforme. Technol Cancer Res Treat. 2004;3:41-9.

11. Sultanem K, Patrocinio H, Lambert C, Corns R, Leblanc R, Parker W, et al. The use of hypofractionated intensity-modulated irradiation in the treatment of glioblastoma multiforme: preliminary results of a prospective trial. Int J Radiat Oncol Biol Phys. 2004;58:247-52.

12. Nwokedi EC, DiBiase SJ, Jabbour S, Herman J, Amin P, Chin LS. Gamma knife stereotactic radiosurgery for patients with glioblastoma multiforme. Neurosurgery. 2002;50:41-6 discussion 46-7.

13. Tanaka M, Ino Y, Nakagawa K, Tago M, Todo T. High-dose conformal radiotherapy for supratentorial malignant glioma: a historical comparison. Lancet Oncol. 2005;6:953-60.

14. Cardinale R, Won M, Choucair A, Gillin M, Chakravarti A, Schultz C, et al. A phase II trial of accelerated radiotherapy using weekly stereotactic conformal boost for supratentorial glioblastoma multiforme: RTOG 0023. Int J Radiat Oncol Biol Phys. 2006;65:1422-8.

15. Laprie A, Pirzkall A, Haas-Kogan DA, Cha S, Banerjee A, Le TP, et al. Longitudinal multivoxel MR spectroscopy study of pediatric diffuse brainstem gliomas treated with radiotherapy. Int J Radiat Oncol Biol Phys. 2005;62:20-31.

16. Ken $S$, Vieillevigne $L$, Franceries $X$, Simon $L$, Supper $C$, Lotterie J-A, et al. Integration method of 3D MR spectroscopy into treatment planning system for glioblastoma IMRT dose painting with integrated simultaneous boost. Radiat Oncol. 2013;8(1).

17. Stupp R, Mason WP, van den Bent MJ, Weller M, Fisher B, Taphoorn MJB, et al. Radiotherapy plus concomitant and adjuvant Temozolomide for glioblastoma. N Engl J Med. 2005;352:987-96.

18. Brandsma D, Stalpers L, Taal W, Sminia P, van den Bent MJ. Clinical features, mechanisms, and management of pseudoprogression in malignant gliomas. Lancet Oncol. 2008:9:453-61.

19. Albert NL, Weller M, Suchorska B, Galldiks N, Soffietti R, Kim MM, et al. Response assessment in neuro-oncology working group and European Association for Neuro-Oncology recommendations for the clinical use of PET imaging in gliomas. Neuro-Oncology. 2016;18:1199-208.

20. Lips IM, van der Heide UA, Haustermans K, van Lin EN, Pos F, Franken SP, et al. Single blind randomized phase III trial to investigate the benefit of a focal lesion ablative microboost in prostate cancer (FLAME-trial): study protocol for a randomized controlled trial. Trials. 2011;12:255.

21. Oehlke O, Mix M, Graf E, Schimek-Jasch T, Nestle U, Götz I, et al. Amino-acid PET versus MRI guided re-irradiation in patients with recurrent glioblastoma multiforme (GLIAA) - protocol of a randomized phase II trial (NOA 10/ARO 2013-1). BMC Cancer. 2016;16:769.

22. Fleckenstein J, Hellwig D, Kremp S, Grgic A, Gröschel A, Kirsch C-M, et al. F-18-FDG-PET confined radiotherapy of locally advanced NSCLC with concomitant chemotherapy: results of the PET-PLAN pilot trial. Int J Radiat Oncol. 2011;81:e283-9.

23. Welz S, Mönnich D, Pfannenberg C, Nikolaou K, Reimold M, La Fougère C, et al. Prognostic value of dynamic hypoxia PET in head and neck cancer: results from a planned interim analysis of a randomized phase II hypoxiaimage guided dose escalation trial. Radiother Oncol. 2017. 
24. Even AJG, Reymen B, La Fontaine MD, Das M, Jochems A, Mottaghy FM et al. Predicting tumor hypoxia in non-small cell lung cancer by combining CT, FDG PET and dynamic contrast-enhanced CT. Acta Oncol (Madr). 2017; 56:1591-6.

25. Carrie C, Grill J, Figarella-Branger D, Bernier V, Padovani L, Habrand JL, et al. Online quality control, hyperfractionated radiotherapy alone and reduced boost volume for standard risk medulloblastoma: long-term results of MSFOP 98. J Clin Oncol. 2009;27:1879-83.

26. Peters L, O'Sullivan B, Giralt J, Fitzgerald TJ, Trotti A, Bernier J, et al. Critical impact of radiotherapy protocol compliance and quality in the treatment of advanced head and neck Cancer: results from TROG 02.02. J Clin Oncol. 2010;28:2996-3001.

27. Ohri N, Shen X, Dicker AP, Doyle LA, Harrison AS, Showalter TN Radiotherapy protocol deviations and clinical outcomes: a meta-analysis of cooperative group clinical trials. JNCI J Natl Cancer Inst. 2013;105:387-93.

28. Weber DC, Tomsej M, Melidis C, Hurkmans CW. QA makes a clinical trial stronger: evidence-based medicine in radiation therapy. Radiother Oncol. 2012;105:4-8.

29. Khalifa J, Tensaouti F, Chaltiel L, Lotterie J-A, Catalaa I, Sunyach MP, et al. Identification of a candidate biomarker from perfusion MRI to anticipate glioblastoma progression after chemoradiation. Eur Radiol. 2016;26:4194-203.

30. Khalifa J, Tensaouti F, Lotterie J-A, Catalaa I, Chaltiel L, Benouaich-Amiel A, et al. Do perfusion and diffusion MRI predict glioblastoma relapse sites following chemoradiation? J Neuro-Oncol. 2016;130.

31. Deviers A, Ken S, Filleron T, Rowland B, Laruelo A, Catalaa I, et al. Evaluation of the lactate-to-N-acetyl-aspartate ratio defined with magnetic resonance spectroscopic imaging before radiation therapy as a new predictive marker of the site of relapse in patients with glioblastoma multiforme. Int J Radiat Oncol Biol Phys. 2014;90:385-93.

Ready to submit your research? Choose BMC and benefit from:

- fast, convenient online submission

- thorough peer review by experienced researchers in your field

- rapid publication on acceptance

- support for research data, including large and complex data types

- gold Open Access which fosters wider collaboration and increased citations

- maximum visibility for your research: over $100 \mathrm{M}$ website views per year

At $\mathrm{BMC}$, research is always in progress.

Learn more biomedcentral.com/submissions 